\title{
Preface: Expertisen und Raumpolitiken: Ein vernachlässigtes Forschungsfeld?
}

\author{
Anne Vogelpohl ${ }^{1}$ and Jeannine Wintzer ${ }^{2}$ \\ ${ }^{1}$ Institut für Geographie, Universität Hamburg, Bundesstr. 55, 20146 Hamburg, Germany \\ ${ }^{2}$ Geographisches Institut, Universität Bern, Hallerstrasse 12, 3012 Bern, Switzerland \\ Correspondence to: Anne Vogelpohl (anne.vogelpohl@uni-hamburg.de) and \\ Jeannine Wintzer (wintzer@giub.unibe.ch) \\ Published: 3 May 2017
}

\section{Expertisen und Raumpolitiken: Einleitung}

Fachgutachten, Diagnosen, Szenarien und Empfehlungen für städtische, regionale oder auch organisatorische Kontexte werden zunehmend durch externe Beratungsinstitute vorgenommen. Damit rücken neue Akteure in das Feld der geographischen Wissensproduktion und raumstrategischen Entscheidungen. Sie nehmen mittels Zahlen, Daten, Graphiken und Prognosen Einfluss darauf, welches Wissen über Räume anerkannt wird und welche Veränderungen als denkbar bzw. nicht-denkbar gelten. Dies materialisiert sich in räumlichen Leitbildern für Stadt und Land, in aus demographischen Prognosen abgeleiteten Bevölkerungspolitiken oder in Bewertungen von Landnutzungen und Ressourcenpotenzialen. Wenig reflektiert bleiben dabei der Prozess der Erkenntnisgewinnung durch die Expert_innen und die damit einhergehende praktisch relevante Auswahl von potenziell machbaren respektive nicht umsetzbaren Möglichkeiten.

Das Themenheft „Expertisen und Raumpolitiken Zur Bedeutung externer Beratung in der geographischen Wissensproduktion" verstehen wir als Anstoß für eine bisher zögerlich geführte Debatte um Zusammenhänge zwischen Beratung, ausgelagerten Fachkompetenzen sowie deren Konsequenzen für politische Entscheidungen in der Stadt- und Regionalentwicklung. Boltanski und Chiapello (2006) betonen in ihrem Buch über den neuen Geist des Kapitalismus, dass heute neben Managern und Coaches auch die Figur des Experten wichtig geworden ist; allerdings haben Pautz (2012) und Prince (2012) darauf hingewiesen, dass die $\mathrm{Zu}$ nahme von Expertisen in der Politikgestaltung bisher kaum empirisch untersucht wurde. Diese Zunahme zeigt sich in Anfragen politischer Parteien sowie Jahresberichten von Behörden und Anstalten des öffentlichen Rechts (z.B. Abge- ordnetenhaus Berlin, 2013; Bürgerschaft der FHH, 2016), aber auch in konzeptionellen Beiträgen zu Geographie und Politik unter Bedingungen global vernetzter Informationen (Larner, 2002; McCann und Ward, 2011; Peck und Theodore, 2010; Volkmann, 2012; s. auch Hurl, 2017; Vogelpohl, 2017 in diesem Heft). Während aber über einen Managerialism in räumlichen Prozessen oder Coaching im Sinne von (Selbst)Optimierung schon viel gesagt wurde, sind wissenschaftliche Analysen über die vielseitigen Formen von Expertisen und deren Einfluss auf politische Entscheidungen noch sehr vereinzelt. ${ }^{1}$

Es gibt bislang kaum Untersuchungen zum Umgang von Expert_innen und Beratenen mit Nicht-Wissen, Unsicherheiten oder expliziter Ausblendung bestimmter Erkenntnisse. Es gibt kaum Langzeitanalysen, die Veränderungen in der Beauftragung von und im Umgang mit Expertisen feststellen könnten oder die die verschlungenen Wege nachvollziehen, die Wissen zwischen Beratung und Politik nehmen kann. Und es gibt kaum Studien, die auf einer intensiven teilnehmenden Beobachtung des Beratungsprozesses oder der Institutionen von Expert_innen einerseits und den politischen Einheiten, die beraten werden, andererseits beruhen. Zudem sind viele Fragen zur Brauchbarkeit von Erfahrungen aus älteren Projekten für neue Probleme, zum Spannungsfeld von Expertise und Partizipation und zur Möglichkeit, dass Expertisen nicht immer nur Lösungen beschleunigen sollen, sondern auch manchmal gerade für eine Verzögerung gesucht werden, weiterhin offen. $\mathrm{Zu}$ diesbezüglichen Forschungen

\footnotetext{
${ }^{1}$ Aktuell läuft allerdings ein Call for Papers der Zeitschrift Peripherie zum Thema „Macht und Prognose“, http://www. zeitschrift-peripherie.de/CfP_Macht-und-Prognose.pdf, letzter Zugriff: 1 März 2017.
} 
soll das vorliegende Themenheft anregen und Perspektiven, Begrifflichkeiten und empirische Ansatzpunkte liefern.

Das Themenheft eröffnet Einblicke in die noch schwach untersuchten Zusammenhänge von Expertisen und Raumpolitiken. Mit der Breite der empirischen Beispiele und der Tiefe der einzelnen Analysen zielt das Heft darauf, die Vielgestaltigkeit des Phänomens zu betonen. Dabei wollen wir das idealtypische Bild von Expert_innen mit Analysen zu den teils konfliktgeladenen politischen Aushandlungsprozessen vor, während und nach der Beratung durch Expertisen sowie mit Analysen zu den institutionellen Hintergründen der Expert_innen und der daraus folgenden Verzerrung der Beratungsinhalte konfrontieren. Gleichzeitig macht die Heterogenität der hier versammelten Beiträge aber auch deutlich, dass die Forschung zu Expertisen und Raumpolitiken erst im Entstehen ist und auf keinen wissenschaftlichen Kanon zurückgreifen kann. Die Beiträge des Themenheftes gehen auf die gleichnamige Fachsitzung auf dem Deutschen Kongress für Geographie in Berlin 2015 zurück. Sie basieren auf unterschiedlichen Begriffen von Expertise, thematisieren verschiedene Politiken, adressieren verschiedene Scales in unterschiedlichen Regionen und verweisen auf ein vielseitiges Methodenrepertoire. Diese Heterogenität zeigt die Breite möglicher Vertiefungen. Gleichzeitig fordert sie zu einer intensiveren Reflexion der Zusammenhänge von Expertise und Raumpolitik auf. Im Folgenden werden die Erkenntnisse des Themenheftes entlang der Aspekte „Expertisen: Formen und Akteure“, „Raumpolitiken: Scales und Regionen“ und „Methoden: Analysen und Zugänge" herausgearbeitet. Dabei thematisieren wir in jedem Kapitel die „Lücken“, die auch mit den vier Beiträgen noch nicht bearbeitet, geschweige denn geschlossen werden können. Auf diese Weise möchten wir erste Konturen am Nexus von Expertisen und Raumpolitiken identifizieren und gleichzeitig Anregungen für zukünftige Studien in diesem Feld bieten.

\section{Expertisen: Formen und Akteure}

Politikberater und Experten sind die Leute, auf die heute viele setzen - und auf die jeder schimpft (Leggewie, 2007:7).

Das Zitat des Politikwissenschaftlers Claus Leggewie ist nach wie vor eine aktuelle Einschätzung zur Bedeutung von Experten (und Expertinnen) in der Politikgestaltung: einerseits werden sie immer häufiger in die Politikgestaltung eingebunden, andererseits wird ihre Rolle häufig als problematisch gesehen. So schaffen es negative Beispiele meist schneller in die öffentlichen Medien wie z.B. überteuerte Beratungsprojekte oder Fehlprognosen (z.B. Berg et al., 2004; Leif, 2006). Solche Projekte mit nur wenig oder ohne Nutzen rufen folgende Fragen hervor: Wie werden externe Expert_innen legitimiert, Politiken relativ direkt zu beeinflussen, obwohl dafür eigentlich Regierungen gewählt werden? Wie werden die hohen Kosten externer Expertisen begrün- det? Warum haben traditionelle Verwaltungen nicht mehr die Kapazitäten und Fähigkeiten, selbst die Politikformulierung zu unterstützen und die Ergebnisse umzusetzen? Wie können einseitige Sichtweisen in der Politikgestaltung auf Grund der spezifischen Fachkompetenz von Expert_innen verhindert werden? Wie kann die Profitorientierung eines privaten Beratungsinstitutes mit der Themenorientierung staatlicher Institutionen in Einklang gebracht werden?

Idealtypisch gelten Expert_innen als neutral, sachkundig, eindeutig und erfahren. Mit diesen Zuschreibungen erscheinen Expert_innen als Gegengewicht zu postfaktischen Vorwürfen. Nicht Emotionalität, sondern Tatsachen, nicht interessengeleitete, sondern validierungsfähige Argumente sollen das Handeln von Expert_innen bestimmen. Ihre Funktion ist ebenso idealtypisch: Sie sollen Entscheidungen vorbereiten, aber nicht treffen und auch nicht umsetzen (Boltanski und Chiapello, 2006; Lendi, 2005; Volkmann, 2012). Dabei sind Gutachten das häufigste Medium von Expertisen. Ein empirischer Blick in Fallbeispiele zeigt allerdings, dass sich in konkreten Projekten eindeutige Zuschreibungen von Expert_innen einerseits und Ratsuchenden andererseits ambivalenter und komplexer darstellen. In dem vorliegenden Themenheft werden hybride Wissensformen, die klare Unterscheidungen zwischen global agierenden „Expert_innen“" und lokalen „Laien“ verunmöglichen (Henrizi, 2017), ebenso herausgearbeitet wie die Problematik der kurzen Dauer eines Beratungsprojektes für mittelfristige Politikvorhaben (Vogelpohl, 2017). Zudem interessiert sich das Heft für die Reduktion des Städtischen auf Input-Output-Fragen unter der einseitigen Maxime der Wirtschaftlichkeit (Hurl, 2017) und wie wissenschaftliche Expertisen zur Legitimation von politischen Strategien argumentativ zum Einsatz kommen (Stenglein, 2017).

Im Zuge des Themenheftes werden NGOs, Unternehmensberatungen, Wirtschaftsprüfungsgesellschaften und Scientific Communities als Akteure angesprochen. Nicht thematisiert werden wissenschaftliche Ressortforschungsinstitute, Universitäten, Planungsbüros, Mediator_innen, Lobbygruppen oder „Gegenexperten“ (Rucht, 1988) wie Personen aus sozialen Bewegungen. Neben diesen noch nicht vertieft untersuchten Expert_innen ist gerade bei Raumpolitiken zudem zu berücksichtigen, dass Partizipation, Teilhabe und Selbstgestaltung wichtige Ziele in Projekten der Stadt- und Regionalentwicklung geworden sind und deshalb auch Bürger_innen explizit als Expert_innen für ihren Lebensalltag in den Räumen einbezogen werden sollten. Gerade die Idee von „Bürger_innen als Expert_innen“ unter dem Begriff „,deliberative Beratung" bzw. „,deliberative Demokratie“ (Martinsen, 2006; Silver et al., 2010) zeigt, dass externe Expertisen in der Regel immer umstritten sind: es gibt Gegengutachten zu Gutachten, es gibt Kritik an Modellen, Grenzwerten oder Aussagen aufgrund von alltäglichen Erfahrungen, die mit den Darstellungen von Expert_innen nicht korrespondieren. Insofern ist der Einfluss von Expertisen auf Räume nicht als eindeutiger, linearer top-down-Prozess zu denken, so dass empiri- 
sche wie konzeptionelle Überlegungen diesen Unterbrechungen Beachtung schenken sollten.

\section{Raumpolitiken: Disziplinen und Scales}

Forschungsfragen zu Expertisen und Raumpolitiken bieten eine relevante Vertiefung für alle Teilbereiche der Geographie. Sie spielen in nahezu jeglichen Subdisziplinen der physischen und Humangeographie eine Rolle und es finden sich empirische Beispiele in allen Regionen der Erde und auf allen Scales - von der Haushaltsebene bis zur Nachbarschaft und dem Dorf bis hin zu global agierenden Netzen wie der $\mathrm{UN}^{2}$ oder nationalstaatlichen Institutionen, die sich für globale Fragen beraten lassen ${ }^{3}$. Aus diesem breitgefächerten Spektrum zur Analyse der Zusammenhänge von Expertisen und Raumpolitiken ergeben sich unserer Ansicht nach zwei Aufgaben: Erstens ist es von Bedeutung danach zu fragen, welche Akteure mit welchen Interessen und Ansätzen in welchen globalen Regionen und auf welchen Scales operieren. Diese Frage impliziert, dass Expert_innen gerade auch dazu beitragen können, das Verständnis einer „Region“ selbst zu verändern oder bestehende skalare Einordnungen zu durchbrechen bzw. erst zu erzeugen (,,jumping scales“, vgl. Smith, 2000). Der Fokus auf die Akteure ermöglicht dabei, die spezialisierten Prozesse der Wissensproduktion und deren Eingang in konkrete Raumpolitiken nachzuvollziehen. Dieser Einfluss kann je nach Interessensstruktur der Expert_innen und ihren Organisationen selbst (z.B. profitorientierten Unternehmensberatungen vs. staatlich geförderten Forschungsinstituten) sowie nach institutionellem Kontext (z.B. mit unterschiedlich ausgeprägten demokratischen Strukturen) stark variieren.

Diese Differenzierung darf jedoch nicht dazu verleiten, die Vernetzung der Akteure und die vielfältigen politischen, wirtschaftlichen und sozialen Beziehungen, die Raumpolitiken prägen, zu vernachlässigen. Deswegen ist es zweitens wichtig, auch die von problem- und ortsspezifischen Expertisen angeleiteten Raumpolitiken in ihrer Relationalität zu begreifen (s. z.B. Peck und Theodore, 2015; Pierce et al., 2011). Konkret bedeutet das, die Situiertheit der konkreten Fälle mit räumlichen Prozessen auf verschiedenen Scales zu verbinden und das Expert_innenwissen in Form von Gutachten, Studien oder Szenarien mit der Positionalität der Expert_innen zu verknüpfen. Erst mit der relationalen Öffnung des Blicks auf das Agieren von Expert_innen werden die strukturellen Rahmenbedingungen expertisengeleiteter Raumpolitiken sichtbar.

Dieses Themenheft spiegelt das breite Spektrum von der Forschung zugrunde gelegten Scales und Regionen bereits wider. Die vier Artikel greifen Entwicklungen in vier Re-

\footnotetext{
${ }^{2}$ https://sustainabledevelopment.un.org/partnership/?p=9691, letzter Zugriff: 7. März 2017.

${ }^{3}$ http://www.wbgu.de/hauptgutachten/, letzter Zugriff: 7 März 2017.
}

gionen auf drei Kontinenten auf - von China im Osten über den Irak und Deutschland bis nach Kanada im Westen. Sie thematisieren Scales von Städten (Hurl, 2017; Vogelpohl, 2017), Regionen (Stenglein, 2017) sowie lokale Wissensströme und ihre globalen Verflechtungen (Henrizi, 2017). Dies unterstreicht, dass das Thema „Expertisen und Raumpolitiken“ nicht nur in westlich geprägten Ländern an Bedeutung gewonnen hat und nicht ausschließlich als dominantes Wissen des ,globalen Nordens“ markiert ist. Wenngleich die hier getroffene Auswahl auch klein ist, so vermag sie im Querschnitt zu zeigen, dass Expertisen Einfluss gewinnen, aber umstritten bleiben und dass das angebotene Wissen vielfältig mit anderen Formen des Erfahrungswissens verknüpft wird.

Wünschenswert sind Studien aus weiteren Subdisziplinen der Geographie über verschiedenste Regionen der Erde und Studien, die entweder international vergleichend sind oder die Multiskalarität räumlicher Prozesse in den Mittelpunkt stellen. Nur so kann eine generalisierbare Typologie von Expertisen, Beratung und Raum an Kontur gewinnen. Und eine solche Typologie könnte helfen, die Machtverhältnisse zu erkennen, die bestimmen, unter welchen Umständen wessen Wissen in der Raumproduktion zur Geltung kommen kann und wessen Wissen marginalisiert wird (vgl. Whatmore, 2009). Dies geht letztlich auch mit methodischen Herausforderungen einher, die wir im Folgenden reflektieren möchten.

\section{4 Überblick über das Heft: Zugänge und Analysen}

Eine Analyse der Produktion, Vermittlung und Durchsetzung von Expert_innenwissen setzt als erstes voraus, dass sowohl die Akteure, also die Expert_innen, als auch das Wissen, das als Expert_innenwissen verstanden wird, identifiziert werden können. Die Zuschreibung „Expert_in“ basiert auf der Vermutung, dass diese Person über exklusives Wissen respektive über Sonderwissen verfügt. Dies trifft auch auf Spezialist_innen zu. Sie besitzen - idealtypisch - jedoch nicht die Autorität, ihre Kompetenzen zu vermitteln und in praktisches Handeln umzusetzen, das heißt diese für Problemlösungen einbringen zu können. Für Hitzler et al. (1994) ist dies eine zentrale Eigenschaft von Expert_innen: sie besitzen eine ,institutionalisierte Kompetenz zur Konstruktion von Wirklichkeit“" (ebd.:25). Das scheint auf den ersten Blick spitzfindig, jedoch ermöglicht diese Differenzierung, dass auch Personen als Expert_innen erkannt werden, die bei Problemlösungen eher über laienhaftes Alltagswissen verfügen. Entscheidend ist, dass sie Wissensbestände nicht nur produzieren, sondern die Autorität besitzen, diese zu vermitteln und an deren Durchsetzung beteiligt sind. Wie gelingt der Zugang zu Expert_innen und zu Expert_innenwissen? Wie kann analytisch nachvollzogen werden, wie Expert_innen ihr Wissen einbringen und zirkulieren lassen? Das Themenheft gibt hier verschiedene Einblicke. 
Chris Hurl interessiert sich in seinem Beitrag (Dis)Assembling policy pipelines: unpacking the work of management consultants at public meetings für die Entstehung, Zirkulation und Durchsetzung von Beratung. Basierend auf der Feststellung, dass diese drei Prozesse der Wissensetablierung dynamische Prozesse darstellen, deren Analyse Akteure, Texte und Technologien sowie die Vielzahl von Standorten auf allen Maßstabsebenen einbinden müssen, entwirft Hurl ein methodisches Raster, das drei analytische Ebenen umfasst: (1) Akteure, die Wissen für die Politikberatung generieren wie Consultants und Wirtschaftsprüfungsgesellschaften, (2) Prozesse der Wissensvermittlung durch Evaluationen und Audits, (3) Durchsetzung von Wissen, was sich in verschriftlichten Empfehlungen, aber auch der Kritik an diesen manifestiert. Grundlage seiner empirischen Studie sind die Empfehlungen von KPMG für Einsparpotenziale der Stadt Toronto in Kanada, die städtische Ausgaben identifizieren und mit Bewertungen nach nötig und nicht-nötig eine ganz bestimmte Vorstellung von Stadt entwerfen. Hurl verfolgt vor allem die Rezeption der fachlichen Wissensproduktion und geht der Frage nach: An welchem Punkt der Wissenszirkulation können sich einige Gruppen mit anderem Wissen durchsetzen? Nicht zuletzt zeigt Hurl, wie der komplexe Prozess der Wissensproduktion, -vermittlung und -durchsetzung räumlich verortet werden kann. Dabei sind es nicht unbedingt die Akteure, die sich durch unterschiedliche Kontexte und Standorte bewegen, als vielmehr die Technologien, die von Wirtschaftsprüfer_innen in die Administrationen von Städten, Regionen und Ländern gelangen.

Annika Henrizi befasst sich in ihrem Beitrag Externe Expertise und lokale Wirklichkeit - Ambivalenzen des lokalglobal-Paradigmas im Irak am Beispiel irakischer Frauenorganisationen mit den Konsequenzen des Handelns internationaler Organisationen auf lokale Gegebenheiten. Als empirisches Beispiel dienen ihr irakische Frauenorganisationen, die in der Hoffnung auf Verbesserung der Lebensbedingungen irakischer Frauen nach dem Sturz Saddam Husseins 2003 enttäuscht wurden. Irakische Frauen können nicht von dem Machtwechsel profitieren; ganz im Gegenteil ist eine Verschlechterung der Sicherheit und der persönlichen Entfaltungsmöglichkeiten von Frauen im Irak zu beobachten. Henrizi stellt sich in Folge dessen die Frage, wie sich irakische Frauenorganisationen gegenüber dem externen Handeln von UN-Organisationen sowie (inter)nationalen Nichtregierungsorganisationen positionieren. Mit dem Ziel die Denkund Handlungsweisen von zivilgesellschaftlichen Frauenorganisationen im Irak zu untersuchen führt Annika Henrizi 29 Interviews und eine Gruppendiskussion mit irakischen Aktivistinnen aus sechs Organisationen durch. Um die Verlinkung externer Expertisen mit lokalen Gegebenheiten untersuchen zu können, werden Organisationen gewählt, die mit internationalen Geldgebern und/oder NGOs zusammenarbeiten. Das Datenmaterial wird durch NGO-Berichte und Statements sowie eigene Aufzeichnen in Folge von teilnehmender
Beobachtung bei Konferenzen, Workshops, informellen Gesprächen und Social-Media-Einträgen ergänzt. Für die Analyse bedient sich Henrizi der Analyseschritte der Grounded Theory: offenes, selektives und axiales Kodieren. Ziel dessen ist es, durch Rekonstruktion der Denk- und Handlungsweisen zur Verdichtung von Aussagen zu kommen, die das Verhältnis von lokalen und externen Akteuren sowie die Positionierung der Frauenorganisationen gegenüber externen Akteur_innen widerspiegelt.

Anne Vogelpohl adressiert in ihrem Beitrag Consulting completed: temporal aspects of expertise in urban development during times of fast policies das Spannungsfeld zwischen kurzzeitiger Beratung und dem langwierigen, komplexen Prozess der Politikgestaltung. Im Fokus stehen die global agierenden Unternehmensberatungen McKinsey und Roland Berger sowie sechs Fälle, in denen diese ihre Beratung eingebracht haben. Sie führt eine Dokumentenanalyse von Beratungs- und Strategiepapieren sowie 41 Interviews mit Personen aus Beratung, Politik, Verwaltung, Kritiker_innen und jenen durch, die direkt an der Umsetzung von der durch Beratung entstandenen Projekte beteiligt waren. Die Analyse der Interviews ist an der dokumentarischen Methode (Bohnsack et al., 2013) orientiert und fokussiert auf die Ausarbeitung von impliziten Meinungen und Überzeugungen der Expert_innen. Mit einer Auswahl von sechs Fallstudien zeigt sie zwei zentrale Dimensionen von Zeitlichkeit auf, die Stadtentwicklung auf Basis von Beratung entscheidend prägen: die kurze Dauer der eigentlichen Beratungsprojekte sowie den Moment, an dem die Beratung abgeschlossen ist und die Ergebnisse in die lokalen Politikabläufe eingespeist werden. Damit vertieft Vogelpohl konzeptionell die Zusammenhänge zwischen global zirkulierenden Politikmodellen, die aufgrund ihrer leicht verfügbaren Modellhaftigkeit heute als „fast policy“ gefasst werden, und der Funktion von Expert_innen in der lokalen Politik. Weiter zeigt Vogelpohl insgesamt auf, dass fast policy, die beschleunigte städtische Politikgestaltung, sowohl eine Modellorientierung als auch eine Professionalisierung von Politikabläufen umfasst - beide auf Kosten einer thematischen Engführung auf wirtschaftspolitische Themen und zu Lasten einer breitgefächerten Stadtentwicklung. Beratungsgeleitete Stadtentwicklung impliziert, so Vogelpohl, einen Ausschluss von sozialen und ökologischen Themen sowie einen Ausschluss von Bürger_innen wie lokalen Initiativen aus der Politikgestaltung.

Ferdinand Stengleins Beitrag zum Thema Wissenschaftliche Expertise und die (Re)Produktion gesellschaftlicher Verhältnisse - Eine argumentationstheoretische Analyse von Diskursen der Ökologie- und Umweltforschung im westlichen China beschäftigt sich mit der Durchsetzung von Wissen und konzentriert sich auf methodische Möglichkeiten, diese diskursiv zu erschließen. Er untersucht wie Scientific Communities durch Argumentation Überzeugung generieren. Stenglein wählt für seine Analyse eine politisch umkämpfte und durch soziale Spannungen geprägte Region im Westen Chinas. Die chinesische Zentralregierung rief 1999 
die Great Western Development Strategy (GWDS) aus, in deren Folge umfangreiche staatlich finanzierte Umweltsanierungsprogramme initiiert wurden. Der Anlass für die Regierung Chinas war, dass der Westen des Landes erstens wirtschaftlich hinter die küstennahen und exportorientierten östlichen Regionen zurückfällt und als Peripherie mit geringer Bedeutung wahrgenommen wird. Zweitens kommt es immer wieder zu gewaltsamen Zusammenstößen zwischen Angehörigen der han-chinesischen, tibetischen und uighurischen Bevölkerung und dem Polizei- und Militärapparat der Volksrepublik China. Und drittens finden massive Umsiedlungs-, Infrastruktur und Investitionsprogramme der Regierung statt, die sowohl die soziale als auch materielle Struktur der westlichen Regionen nachhaltig verändern. In diesen Kontexten arbeitet die Ökologie- und Umweltforschung und zwar zumeist in der Art, dass sie die Konstruktionen der chinesischen Regierungen schlicht reproduzieren. Stenglein untersucht über 100 wissenschaftliche Publikationen dieser Scientific Community aus den Jahren 1999 bis 2013 mittels der Argumentationsanalyse von Stephen Toulmin (2003 [1958]) im Hinblick auf deren diskursiven Entfaltung räumlicher Repräsentationen sowie die Etablierung spezifischer Wissensordnungen. Dabei verweilt Stengleins Analyse nicht auf der bloßen Beschreibung des Argumentationsprozesses und dessen Aufschlüsselung. Da er Argumente als Versprachlichung gesellschaftlicher Diskurse versteht, gelingt ihm die Fruchtbarmachung der Argumentationsanalyse als Diskursanalyse und damit die Analyse der Produktion und Stabilisierung von Wissensordnungen durch Scientific Communities. Er zeigt, dass die Argumentationen im Wesentlichen auf der Repräsentation von ,guten“ und ,schlechten“ Subjekten und Praktiken beruhen. Damit werden einerseits die territoriale Kontrolle des chinesischen Nationalstaats stabilisiert und andererseits die han-chinesischen Eliten legitimiert. Mit diesen Ergebnissen wird deutlich, dass wissenschaftliches Argumentieren und dessen Einsatz als wissenschaftliche „Evidenz" überaus wirkmächtig ist und mit ihnen Möglichkeiten der Machterhaltung aufrechterhalten werden.

Die vier Beiträge des Themenheftes präsentieren Praktiken von Expert_innen als über Sprache, Netzwerke und institutionelle Rahmenbedingungen vermittelt. Die Analyse des Expert_innenwissens erfolgt anhand von Interviews, Berichten, Protokollen, Social-Media-Einträgen und wissenschaftlichen Beiträgen. Aus der Perspektive des Linguistic Turns in der Geographie (vgl. Wintzer und Wastl-Walter, 2016) betrachtet, lässt sich verallgemeinert sagen, dass das (Expert_innen-)Wissen über die Welt nicht per se vorhanden ist, sondern durch sprachliche Interaktion von Akteur_Innen hergestellt und aktualisiert wird. Der Linguistic Turn könnte auch zukünftig in der Geographie eine Abwendung von realistischen Auffassungen hin zu den sprachlichen Leistungen der Akteur_innen ermöglichen. Wichtig ist jedoch, dass nicht-sprachliche Konstruktionsprozesse ebenso wissenschaftliche Beachtung finden (vgl. Lossau, 2008). Diese betonen, dass Praktiken von der Sprache über Mimik und
Gestik bis hin zu performativen Prozessen der Körperlichkeit reichen und in der Empirie Beachtung finden müssen. Im Zuge dessen könnte eine geographische Expertisenforschung die Inszenierungen von Autorität sowie soziale und kulturelle Kapitalsorten (wie zum Beispiel persönliche Netzwerke von Stakeholdern, die neben wirtschaftlichem Kapital eine bedeutende Rolle bei der Vermittlung und Durchsetzung von (geographischen) Wissen über Raum spielen) intensiver beleuchten.

In den Beiträgen des Themenheftes ist viel zu Expert_innenwissen zu lesen, jedoch fehlt es der Beleuchtung von Nichtwissen beziehungsweise zu Phänomen des ,nichtwissen-wollen“. Die Beiträge verweisen einerseits auf die Produktion, Vermittlung und Durchsetzungsstrategien von Expert_innenwissen, andererseits reflektieren sie diese Praktiken als machtvolle Instrumente der Wirklichkeitskonstruktion. In der geographischen Forschung zu Wissensproduktionen erlangen jedoch zunehmend Untersuchungen zu Ignoranz, Wissenslücken und Nichtwissen an Bedeutung. Wichtig wäre es in dem Zuge, die bewusste Ausblendung störender Sachverhalte, also eine strategische Komplexitätsreduktion, zu betrachten und zu analysieren, wie gerade durch sie wirtschaftlicher Erfolg und politische Wirksamkeit erzielt wird. Erst die Leugnung komplexer Verursachungszusammenhänge macht eine schnelle und auf Lösungen ausgerichtete Beratung möglich. Fragen danach, wie Nichtwissen empirisch erhoben und ausgewertet werden kann, wie der empirische Umgang mit Ignoranz gelingen kann und wie die damit einhergehenden Machtverhältnisse rekonstruiert werden können, bleiben bisher unbeantwortet.

\section{Literatur}

Abgeordnetenhaus Berlin: Kleine Anfrage der Abgeordneten Susanne Graf (PIRATEN) vom 28. Februar 2013 und Antwort, Betr.: Schlecht beraten Berlin?, Drucksache 17/11652, 2013.

Berg, S., Fröhlingsdorf, M., Kurz, F., Latsch, G., Meyer, C., und Schumann, H.: Im Reich der Träume, Der Spiegel, 2004, 60-65, 2004.

Bohnsack, R., Nentwig-Gesemann, I. und Nohl, A.-M.: Die dokumentarische Methode und ihre Forschungspraxis - Grundlagen qualitativer Sozialforschung, Springer VS, Wiesbaden, 2013.

Boltanski, L. und Chiapello, Ė.: Der neue Geist des Kapitalismus, UVK, Konstanz, 2006.

Bürgerschaft der FHH (Bügerschaft der Freien und Hansestadt Hamburg): Schriftliche Kleine Anfrage der Abgeordneten Katja Suding (FDP) vom 27.01.16 und Antwort des Senats, Betr.: Beraterverträge/Gutachten; Drucksache 21/3041, 2016.

Henrizi, A.: Externe Expertise und lokale Wirklichkeit - Ambivalenzen des lokal-global-Paradigmas im Irak am Beispiel irakischer Frauenorganisationen, Geogr. Helv., 72, 29-44, doi:10.5194/gh-72-29-2017, 2017.

Hitzler, R., Honer, A. und Maeder, Ch.: Expertenwissen - Die Institutionalisierte Kompetenz zur Konstruktion von Wirklichkeit, Westdeutscher Verlag, Opladen, 1994. 
Hurl, C.: (Dis)Assembling policy pipelines: unpacking the work of management consultants at public meetings, Geogr. Helv., 72, 183-195, doi:10.5194/gh-72-183-2017, 2017.

Larner, W.: Globalization, Governmentality, and Expertise: Creating a Call Centre Labour Force, Rev. Int. Polit. Econ., 9, 650674, doi:10.1080/0969229022000021844, 2002.

Leggewie, C.: Das Ohr der Macht und die Kunst der Konsultation: Zur Einleitung, in: Von der Politik- zur Gesellschaftsberatung. Neue Wege öffentlicher Konsultation, Herausgeber: Leggewie, C., Campus, Frankfurt a. M., New York, 7-13, 2007.

Leif, T.: Beraten \& verkauft: McKinsey \& Co. - der große Bluff der Unternehmensberater, C. Bertelsmann, München, 2006.

Lendi, M.: Politikberatung - Nachfrage, Resonanz, Alibi, vdf Hochschulverlag, Zürich, 2005.

Lossau, J.: Kulturgeographie als Perspektive, Berichte zur Deutschen Landeskunde, 82, 317-334, 2008.

Martinsen, R.: Partizipative Politikberatung - der Bürger als Experte, in: Handbuch Politikberatung, Herausgeber: Falk, S., Rehfeld, D., Römmele, A. und Thunert, M., VS, Wiesbaden, 138-151, 2006.

McCann, E. und Ward, K.: Mobile Urbanism - Cities and Policymaking in the Global Age, University of Minnesota Press, Minneapolis, 2011.

Pautz, H.: Think-Tanks, Social Democracy and Social Policy, Palgrave Macmillan, Basingstoke, New York, 2012.

Peck, J. und Theodore, N.: Mobilizing Policy - Models, Methods and Mutations, Geoforum, 41, 169-174, doi:10.1016/j.geoforum.2010.01.002, 2010.

Peck, J. und Theodore, N.: Fast Policy - Experimental Statecraft at the Thresholds of Neoliberalism, University of Minnesota Press, Minneapolis, London, 2015.

Pierce, J., Martin, D. G. und Murphy, J. T.: Relational Place-Making - The Networked Politics of Place, T. I. Brit. Geogr., 36, 54-70, doi:10.1111/j.1475-5661.2010.00411.x, 2011.
Prince, R.: Policy Transfer, Consultants and the Geographies of Governance, Prog. Hum. Geog., 36, 188-203, doi:10.1177/0309132511417659, 2012.

Rucht, D.: Gegenöffentlichkeit und Gegenexperten: Zur Institutionalisierung des Widerspruchs in Politik und Recht, Zeitschrift für Rechtssoziologie, 9, 290-305, 1988.

Silver, H., Scott, A. und Kazepov, Y.: Participation in Urban Contention and Deliberation, Int. J. Urban Regional, 34, 453-477, doi:10.1111/j.1468-2427.2010.00963.x, 2010.

Smith, N.: Scale, in: The Dictionary of Human Geography, Herausgeber: Johnston, R., Gregory, D., Pratt, G. und Watts, M., Blackwell, Oxford, 724-727, 2000.

Stenglein, F.: Wissenschaftliche Expertise und die (Re)Produktion gesellschaftlicher Verhältnisse - Eine argumentationstheoretische Analyse von Diskursen der Ökologie- und Umweltforschung im westlichen China, Geogr. Helv., 72, 197-209, doi:10.5194/gh-72-197-2017, 2017.

Toulmin, S.: The Uses of Argument, Cambridge University Press, Cambridge, 2003.

Wintzer, J. und Wastl-Walter, D.: Neue Kulturgeographie, in: Sprache - Kultur - Kommunikation / Language - Culture - Communication - Ein internationales Handbuch zu Linguistik als Kulturwissenschaft / An International Handbook of Linguistics as a Cultural Discipline, Herausgeber: Jäger, L., Holly, W., Krapp, P., Weber, S. und Heekeren, S., De Gruyter, Berlin, 283-294, 2016.

Vogelpohl, A.: Consulting completed: temporal aspects of expertise in urban development during times of fast policies, Geogr. Helv., 72, 65-76, doi:10.5194/gh-72-65-2017, 2017.

Volkmann, U.: Reine Vernunft - Zehn Skizzen über den Aufstieg der Experten und den Abschied des Politischen, Merkur - Deutsche Zeitschrift für europäisches Denken, 66, 765-775, 2012.

Whatmore, S.: Mapping Knowledge Controversies: Science, Democracy and the Redistribution of Expertise, Prog. Hum. Geog., 33, 587-598, doi:10.1177/0309132509339841, 2009. 